\title{
Circulation et patrimonialisation des pratiques esthétiques expressives dans le Pacifique : les exemples de Tonga et de la Nouvelle-Zélande
}

\author{
Aurélie Condevaux*1
}

Poser la question du lien entre processus de patrimonialisation et mobilités - en particulier migratoires - ne va pas de soi. La préoccupation pour le « patrimoine » qui s'affirme dans le contexte de construction des États nations au XIX ${ }^{\mathrm{e}}$ siècle est en effet dès le départ fortement ancrée dans un territoire qu'il s'agit de définir [Clavairolle, 2014]. Pourtant, l'expression de «patrimoine de l'immigration », qui s'impose en France avec par exemple la création de la Cité nationale de l'histoire de l'immigration, ou encore celle de «sites transnationaux et transfrontaliers en série » [Unesco, 2010] - définis comme des biens dont la signification dépasse les frontières d'un pays - laissent penser que l'idée selon laquelle la définition du « patrimoine » participe à la fabrication d'appartenances territoriales et sociales localisées mérite d'être complexifiée. Cela apparaît avec une acuité toute particulière dans la région Pacifique, où les migrations constituent sans doute l'un des phénomènes les plus marquants de la reconfiguration sociale au cours des dernières décennies [Chappell, 1999 ; Gershon, 2007 ; Hermann, Kempf, van Meijl, 2014]. En parallèle à la mobilité des acteurs, de nombreuses pratiques chantées et dansées circulent. Ce phénomène, loin d'être nouveau, est exacerbé aujourd' hui par les migrations, mais aussi par le nombre grandissant de festivals et par la mise en tourisme des cultures et des «traditions». Ainsi, au cours de performances touristiques, les visiteurs peuvent découvrir le fameux haka māori de NouvelleZélande à Hawai'i ou à Tonga, alors que les danses de Polynésie française, des Îles Cook ou de Hawai 'i peuvent être exécutées aussi bien à Fidji qu'à Wellington, capitale de la Nouvelle-Zélande.

\footnotetext{
* Anthropologue, maître de conférences, université Paris 1 Panthéon-Sorbonne, EIREST (Équipe Interdisciplinaire de Recherches sur le Tourisme), EA 7337.

1. Je remercie toutes les personnes qui ont contribué à la réalisation des enquêtes sur lesquelles cet article est fondé, et en particulier le département de la recherche du Te Papa et les managers du Tongan National Cultural Center pour avoir autorisé ces recherches.
} 
Dans certains contextes - mais pas tous -, ces pratiques esthétiques se voient attribuer une valeur patrimoniale: comment expliquer cela ? L'association observée entre mise en patrimoine et mobilité migratoire pourrait laisser penser $a$ priori que cette dernière est un facteur de rupture potentiellement propice à la requalification patrimoniale. L'expérience de la migration jouerait ainsi un rôle déclencheur, instillant la «nostalgie de la perte» chez celui ou celle qui quitte son pays natal, l'amenant à porter un regard nouveau sur ces pratiques. Nous verrons que la rupture que constitue la migration ne peut expliquer seule la requalification patrimoniale. D'autres éléments doivent être convoqués à cette fin, en particulier les contextes politiques et les enjeux identitaires qui entourent ces performances.

L'étude des processus de fabrication patrimoniale qui émergent de ces mobilités permet d'aborder deux axes de questionnements : d'une part, si l'on admet que la construction du « patrimoine » est intimement liée à celle des appartenances sociales, l'étude des changements de significations attribuées à ces pratiques esthétiques est un moyen de comprendre en quoi la migration est « une manière d'éprouver ses sentiments et, plus particulièrement, son sentiment d'appartenance » [Fourcade, Legrand, 2008, p. 7]. D'autre part, il s'agira de mettre en lumière ce que l'accès de ces «patrimoines » à l'espace public, leur reconnaissance dans la société d'installation par le biais des institutions, peut nous dire de la place qui est accordée aux migrants et la manière dont cette reconnaissance procède [Bertheleu, 2014].

Les deux contextes qui seront envisagés sont ceux de Tonga et de la NouvelleZélande. Ces deux archipels indépendants de ce qui est communément appelé le «triangle polynésien ${ }^{2}$ présentent des différences importantes tant du point de vue de l'histoire coloniale que de celui des configurations culturelles actuelles [Van der Grijp, 2001]. L'histoire tongienne, depuis les contacts avec les Européens, est généralement perçue comme donnant à voir des ruptures moins brutales que celles qui ont marqué l'histoire de la Nouvelle-Zélande. Tonga a été sous protectorat britannique de 1900 à 1970, mais n'a jamais été directement colonisé, alors que la Nouvelle-Zélande est passée sous souveraineté britannique dès 1840 . Durant les années 1850-1860, la puissance coloniale britannique s'appropria, par ce qui s'apparente plus à des spoliations qu'à des achats en bonne et due forme, la plupart des terres de Nouvelle-Zélande [Kawharu, 1977]. À Tonga au contraire, en mettant en place une constitution qui interdisait la vente des terres en 1875, le roi George Tupou I assurait ainsi que l'archipel ne passerait pas sous contrôle colonial. Aujourd'hui, alors que la population tongienne est très majoritairement

2. On entend communément par Polynésie une aire géographique et culturelle principalement comprise entre Hawai'i, l'île de Pâques et la Nouvelle-Zélande, région qui comprend aussi bien des nations souveraines que des pays sous domination étrangère. Le terme de Polynésie renvoie à une cartographie au caractère racial élaborée par les premiers Européens qui naviguèrent dans le Pacifique, et est généralement défini par opposition à la Mélanésie et à la Micronésie [Tcherkézoff, 2007]. Si tout le monde semble s'accorder aujourd'hui à dire que l'usage du terme Mélanésie est très contestable, en revanche, "il demeure légitime de considérer la Polynésie (...) comme une unité » [Ibid., p. 319]. 
« polynésienne », en Nouvelle-Zélande, d'après le recensement de 2013, les individus qui s'identifient au groupe «New Zealand European» représentaient $68,00 \%$ de l'ensemble des personnes s'identifiant à un groupe ethnique alors que les «Māori » en constituaient 14,9\% ${ }^{3}$ [Statistics New Zealand, 2013].

Malgré ces différences majeures, ces archipels sont liés par le fait qu'ils partagent une histoire préeuropéenne commune (liée au peuplement de l'Océanie) et par les flux migratoires actuels : la majorité des émigrés tongiens - soit 40713 personnes selon Esau et 60336 selon le recensement de 2013 - vivraient en Nouvelle-Zélande, les autres se répartissant principalement entre l'Australie et les États-Unis ['Esau, 2005, p. 443]. Enfin, nombre de styles de danses polynésiennes circulent entre ces deux archipels, ce qui rend leur examen propice pour répondre aux questions soulevées précédemment. Les données mobilisées ici proviennent d'une ethnographie multisituée conduite entre 2008 et 2013 en Nouvelle-Zélande et à Tonga, ethnographie fondée sur l'observation participante et des entretiens.

\section{Contexte : performances musicales et chorégraphiques «polynésiennes»}

Les pratiques esthétiques expressives ${ }^{4}$ des différents archipels du «triangle polynésien » partagent certains points communs : que ce soit à Tahiti, Hawai'i, Tonga ou en Nouvelle-Zélande, elles sont fréquemment accompagnées par des instruments de musique (guitares et ukuleles) et basées sur une poésie chantée qui est «mimée », évoquée ou illustrée par les mouvements de bras et de mains [Kaeppler, 2001, p. 54]. Certaines pratiques chorégraphiques ne sont pas accompagnées de musique instrumentale, mais de percussions rythmées. Bien que l'on puisse envisager ces similarités comme formant un «canon » polynésien spécifique [Ibid.], il existe également des différences régionales significatives. Dans les performances féminines hawaïennes et tahitiennes (hula, 'aparima, 'òte 'a), les mouvements de bras sont accompagnés de mouvements de hanche, qui sont plus ou moins rapides, mais toujours assez amples. À Tonga en revanche, les jeunes femmes exécutant la danse féminine la plus populaire - le tau'olunga - doivent maintenir leurs bustes et leurs hanches soigneusement alignées.

Peut-être plus que les techniques corporelles mobilisées, ce qui apparente les performances musicales et chorégraphiques de Polynésie les unes aux autres est le fait qu'elles sont souvent étroitement liées à l'ancrage territorial des groupes sociaux, ce qui rend l'étude de leur circulation et de leur patrimonialisation tout particulièrement pertinente pour les questions soulevées précédemment. À Tonga, par exemple, chaque village, district ou île a une ou plusieurs compositions

3. Ces chiffres sont calculés à partir de l'auto-identification des personnes recensées, qui peuvent cocher plusieurs des catégories qui leur sont proposées ou indiquer une catégorie non proposée.

4. Je reprends ici l'expression de K. Alexeyeff [2009], qui désigne par là les danses, la musique, la poésie, mais également certaines pratiques d'ornementation corporelle. Cette formulation est plus appropriée aux contextes polynésiens que les termes de «danse » ou de « chant ». 
musicale et chorégraphique qui lui sont propres et qui permettent immédiatement d'identifier l'origine du groupe de danseurs. Les poèmes sont écrits à partir de références métaphoriques aux éléments paysagers les plus significatifs de ces lieux de vie. Les créations chorégraphiques sont en outre l'œuvre d' « experts » appelés punake, qui sont généralement identifiés par leur appartenance à un village ou à une île (ils sont connus pour être les punake de tel village, de tel quartier, etc.). Leurs compositions sont utilisées et exécutées avant tout par les personnes de ce lieu.

En Nouvelle-Zélande, on note de manière similaire un ancrage territorial des pratiques esthétiques puisque celles-ci sont généralement considérées comme la « propriété » du groupe de leur créateur. Ces créations représentent ou symbolisent des appartenances territorialement localisées, ce qui est tout particulièrement visible dans la pratique des haka dans les cérémonies d'accueil (mihi) sur les marae (espaces cérémoniels des villages māori). La réception d'un groupe de « visiteurs » par un groupe «local» (tangata whenua, les «gens de la terre ») passe notamment par la performance d'un haka pōwhiri, qui permet au groupe recevant d'afficher son identité. Ces identités locales sont également exprimées lors de compétitions. La majorité des groupes de compétiteurs sont fondés sur une appartenance de groupe - celle au hapu ou à l'iwi-qui font partie des unités sociales de référence de la société māori : l'appartenance à ces groupes, qui portent le nom d'un ancêtre réel ou mythique, se définit par un lien de descendance en filiation matrilinéaire ou patrilinéaire par rapport à cet ancêtre (cela ne signifie pas nécessairement que l'appartenance au groupe soit déterminée par une filiation réelle, voir par exemple à ce sujet Schwimmer [1990]). Ces liens étroits entre les pratiques esthétiques musicales et chorégraphiques et l'ancrage territorial de leurs chorégraphes/compositeurs et praticiens ont été notés dans d'autres archipels, notamment à Hawai 'i et à Fidji [Torgersen, 2009], et l'on pourrait sans doute étendre le constat au-delà.

Ces ancrages territoriaux sont néanmoins remis en cause par d'importantes circulations [Lawrence, 1992 ; Moulin, 1996 ; Stillman, 1999], qui préexistent à l'arrivée des Européens [Moyle, 1991]. Néanmoins, la colonisation, puis le développement de nouveaux moyens de communication et de transport et les migrations sont venus donner un second souffle à ces mobilités, qui accompagnent celles des acteurs ou sont véhiculées par le biais des nouvelles technologies. À Tonga par exemple, les danses de Polynésie orientale, présentes depuis plusieurs décennies, sont réputées avoir été d'abord introduites par un Tahitien qui a résidé dans l'archipel au moment du règne de la Reine Sālote (1918-1965). Une école de danse hawaïenne et tahitienne a été créée récemment ${ }^{5}$ par une Tongienne ayant vécu à Hawai'i une grande partie de sa vie. L'introduction des kapa haka (arts performatifs des Māori de Nouvelle-Zélande) par l'une des troupes de danseurs se produisant sur les scènes touristiques résulte quant à elle du fait que l'un des

5. Après une première tentative dans les années 1980, celle-ci rouvre dans les années 2000. 
fondateurs du groupe a travaillé plusieurs années en Nouvelle-Zélande. Les vidéos trouvées sur internet sont, au dire des acteurs, un autre médium de la diffusion et de l'apprentissage. D'emblèmes identitaires locaux, ces pratiques musicales et chorégraphiques qui circulent deviennent, dans certains types d'interactions (liées notamment au tourisme ou aux festivals), le lieu où s'affirment des identités plus englobantes, notamment nationales ou « ethniques », mais aussi pan-nationales ou pan-ethniques.

Le sens qui leur est attribué varie néanmoins en fonction des lieux où elles sont exécutées. Ces dernières années, les concepts relatifs au «patrimoine », diffusés par les organismes internationaux comme l'Unesco, ont été appropriés pour qualifier certaines performances : le lakalaka tongien a ainsi été inscrit sur la liste du patrimoine culturel immatériel de l'humanité, alors que ce même terme de « intangible cultural heritage » est utilisé par le gouvernement néo-zélandais pour désigner les kapa haka [voir par exemple ACCU, 2009] ${ }^{6}$. La requalification patrimoniale ne concerne-t-elle que des pratiques considérées comme «locales » ou « nationales » qui seraient seules significatives pour la construction des appartenances sociales ? Les performances qui circulent et qui sont «empruntées » à d'autres archipels peuvent-elles également être mobilisées dans la construction d'identités collectives ?

\section{Tonga : des « Polynésiens » non polynésiens ?}

Tonga, archipel indépendant du Pacifique sud, est réputé être le dernier « royaume vivant» de cette région et également le «berceau de la Polynésie ». Malgré l'émergence d'un mouvement démocratique depuis quelques décennies, le monarque tongien demeure un chef d'État puissant. L'une des grandes caractéristiques de la société tongienne est sa « dispersion » liée à un phénomène massif d'émigration. Le nombre de Tongiens vivant dans les pays du pourtour du Pacifique (Nouvelle-Zélande, Australie, États-Unis) atteindrait, selon certains, le double de celui des habitants du royaume, soit de 200000 à 250000 personnes [Besnier, 2011, p. 42], les données officielles étant considérées comme largement sous-évaluées ['Esau, 2005, p. 443]. De ce fait, loin d'être un archipel isolé du Pacifique, Tonga est amplement «mis en connexion » avec le reste du monde, et en particulier avec les autres migrants du Pacifique.

On compte environ dix genres de faiva fakatonga, c'est-à-dire de performances musicales et chorégraphiques considérées comme spécifiquement tongiennes. La danse et la musique sont omniprésentes à Tonga. Il ne semble pas y avoir d'événement social d'importance sans une performance, en particulier lorsque ces événements concernent la famille royale. Les faiva fakatonga sont d'ailleurs intimement liés à la reproduction de la structure sociale hiérarchique [Kaeppler, 1993].

6. Il est à noter que les représentations endogènes qui entourent ces pratiques ne peuvent être réduites au concept occidental de «patrimoine ». Ce point mériterait une étude à part entière. 
À côté des faiva fakatonga, d'autres types de danses polynésiennes sont pratiqués dans l'archipel, comme évoqué précédemment. 'Aparima ou 'òte 'a tahitien, hula hawaiien, haka māori, ou encore siva samoan peuvent être observés dans des contextes divers : par exemple lors du concours d'élection de la Miss Tonga (Miss Heilala), qui a lieu chaque année au mois de juin ou juillet. Ces genres y ont notamment été introduits par les candidates vivant à l'étranger où celles-ci ont plus facilement accès à des cours de danses hawaïennes ou tahitiennes que tongiennes [Teilhet-Fisk, 1996, p. 193]. Ces autres danses «polynésiennes » sont aussi fréquemment exécutées lors de fêtes scolaires ou religieuses, de fund raisings (collectes de fonds) et, comme évoqué précédemment, une école de danse propose des cours dans ce domaine à Nuku'alofa (capitale de Tonga). Mais les lieux où ces performances non tongiennes sont le plus fréquemment exécutées sont les représentations touristiques, de type « dîner-spectacle », qui sont fréquentées aussi bien par des touristes internationaux que des Tongiens ou touristes d'origine tongienne en visite dans leur pays d'origine. Ces lieux sont des contextes particulièrement propices pour étudier les constructions identitaires, puisqu'ils constituent une « zone frontière », où l'identité se construit en référence à un « autre » [Adams, 2003 ; Doquet, 2003, p. 11 ; Nesper, 2003].

Les performances touristiques qui font le choix de ne présenter que des faiva tongiens sont très largement minoritaires sur l'île de Tongatapu (principale île tongienne), la plupart préférant proposer un «mélange » de ces différentes pratiques expressives polynésiennes. Cette inclusion est généralement justifiée par les managers de ces performances en soulignant que les faiva fakatonga risqueraient d'ennuyer les touristes non Tongiens, car trop lents ou peu dynamiques. Les autres pratiques expressives polynésiennes - en particulier hawaïennes et tahitiennes sont incluses notamment pour satisfaire le désir de touristes en quête d'une image stéréotypée de la femme polynésienne, libre et sensuelle [Stillman, 1988]. Quelle que soit la justification de leur inclusion, il est en tout cas remarquable de noter que les danses polynésiennes non tongiennes ne sont jamais le support d'un discours identitaire pan-polynésien et qu'elles ne sont en rien définies comme un «patrimoine» commun. Cela est perceptible tout autant dans les discours qui entourent ces performances dans leur contexte d'exécution, que dans les propos recueillis au cours d'entretiens avec les danseurs ou managers de ces centres [Condevaux, 2011].

Du point de vue des jeunes femmes participant à ces performances, la culture tongienne est très différente des autres cultures «polynésiennes » [Ibid.]. Les danses sont d'ailleurs considérées comme emblématiques de cette différence, qui est exprimée dans le langage : les danses tongiennes sont appelées faiva fakatonga, alors que les autres sont toutes rassemblées dans la catégorie faiva fakapolinisia (littéralement : performances musicales et chorégraphiques polynésiennes ou «à la manière » des Polynésiens). Selon cette catégorisation, Tonga est donc placé hors de cet espace polynésien largement construit par le regard européen. Malgré cette introduction de danses venues d'ailleurs, les acteurs résistent à l'imposition d'une identité « polynésienne » et réaffirment leur identité nationale, comme c'est 
le cas dans d'autres contextes voisins concernés par ces circulations [Mageo, 2008].

De plus, seuls les faiva fakatonga font l'objet d'une reconnaissance institutionnelle de type patrimonial à Tonga. Cette reconnaissance prend plusieurs formes. Elle passe d'abord par l'intermédiaire des institutions patrimoniales internationales. Ainsi, comme évoqué précédemment, le lakalaka (discours poétique dansé et chanté, exécuté en groupe) a été choisi par le gouvernement tongien pour une candidature à l'inscription sur la liste des chefs-d'œuvre du patrimoine oral et immatériel de l'humanité de l'Unesco, candidature qui a été retenue en 2003 (cette inscription a par la suite été reportée sur la liste représentative du patrimoine culturel immatériel de l'humanité en 2008).

À l'échelle nationale, les institutions patrimoniales, peu nombreuses, focalisent également leur attention sur les faiva fakatonga. Le Tongan National Cultural Center, qui abrite une petite collection muséale sur l'histoire de l'archipel et qui propose de manière hebdomadaire une performance musicale et dansée de type « dîner-spectacle » similaire à celles évoquées précédemment, mettait un point d'honneur, avant sa privatisation en 2009, à ne présenter que des faiva fakatonga, contrairement à la majorité des autres performances touristiques. Cela répondait aux lignes directrices du musée établies à la fin des années 1990, qui stipulaient que le but de l'institution était notamment « to preserve and portray the culture and traditions of the Tongan Nation and to integrate these for the promulgation of a national identity » [Government of Tonga, 1998, p. 36]. Les personnes travaillant au Centre culturel contrastaient ce travail de sauvegarde avec ce qui est fait au sein des performances touristiques perçues par eux comme de simples lieux de divertissement, bien que les performances dans les deux cas suivent la même forme et soient destinées à des publics similaires. Selon ces acteurs, les autres danses polynésiennes étaient clairement exclues de la «tradition » et du «patrimoine » tongien. Il est en outre significatif que les faiva présentés au Centre Culturel National étaient principalement des compositions de la reine Sālote, réputée être la plus grande punake (expert en composition musicale, poétique et chorégraphique) que Tonga ait connue. Ce choix peut s'expliquer en partie du fait que, par son statut social exceptionnel, la reine était seule autorisée à composer pour toutes les îles et tous les villages de Tonga - et à faire référence à ceux-ci dans ses compositions -. Seules ses œuvres peuvent donc être pleinement considérées comme un «patrimoine » commun à tous les Tongiens, contrairement à celles de punake de statut moins élevé qui, comme évoqué précédemment, dédient leurs compositions à un territoire plus circonscrit.

En même temps, ce choix doit être compris au regard des enjeux politiques sous-jacents à cette entreprise patrimoniale et au cadre dans lequel elle prend place. En 2008, le TNCC était encore sous la tutelle directe de la princesse Sālote Mafile'o Pilolevu Tuita (sœur des rois George Tupou V, qui régnait alors, et Tupou VI, actuellement au pouvoir). Le processus de désignation du «patrimoine » accompagne ici un processus de construction d'une identité nationale, 
dont la monarchie est présentée comme un symbole fort. L'émigration est-elle perçue comme une menace pour la cohésion de la structure sociale hiérarchique et de ce fait pour les « traditions » tongiennes [Johannson Fua et al., 2011, p. 21] ? Comme beaucoup d'entreprises patrimoniales à Tonga, celle du TNCC contribuait à donner une vision autorisée de l'histoire tongienne, largement racontée du point de vue de la famille royale et des nobles. Elle participait à la légitimation de la dynastie au pouvoir, laquelle fait face à un mouvement démocratique depuis quelques décennies. Il est significatif que, depuis sa privatisation en 2009 le Centre Culturel National, qui a conservé ce nom, inclue désormais différents types de danses polynésiennes.

\section{Circulation des pratiques expressives et identité «pan-polynésienne » en Nouvelle-Zélande}

L'introduction de danses venues de divers archipels du Pacifique sud et son lien avec l'émergence possible d'une identité polynésienne se posent d'une manière différente en Nouvelle-Zélande. Les premiers habitants de NouvelleZélande, désignés depuis la colonisation par le terme de Māori, sont arrivés il y a environ 800 ans $^{7}$ depuis, pense-t-on, des îles qui se trouvent aujourd'hui en Polynésie française. Dans la cartographie du Pacifique élaborée par les Européens, les Māori de Nouvelle-Zélande furent rattachés à l'ensemble polynésien [Tcherkézoff, 2007]. Les Māori partagent en effet des traits culturels et linguistiques communs avec les premiers habitants des archipels de l'aire polynésienne, du fait de l'histoire du peuplement de cette région.

La question du caractère «Polynésien » de la Nouvelle-Zélande et de ses habitants est rouverte aujourd'hui par les phénomènes migratoires récents. À partir des années 1950-1960, des migrants venant principalement des Îles Cook, de Tokelau, Niue, Sāmoa et, plus récemment, de Fidji et Tonga, se sont installés en Nouvelle-Zélande [Goss, Lindquist, 2000, p. 392]. Ce mouvement, qui connut un ralentissement à la fin des années 1970, s'est même inversé dans les années 1980, marquées par des migrations en retour [Cook, Didham, Khawaja, 2001]. Ces immigrés et leurs descendants constituent une part non négligeable de la population puisqu'en 2013, 7,4\% des Néo-zélandais s'identifiaient avec un ou plusieurs groupe(s) ethnique(s) du Pacifique [Statistics New Zealand, 2013], contre 6,9\% en 2006. La part grandissante occupée par ce groupe dans la population doit moins aux migrations actuelles qu'à la croissance naturelle et aux changements d'identification [Cook, Didham, Khawaja, 2001, p. 46].

L'arrivée de ces migrants du Pacifique s'est traduite par l'émergence de nouvelles catégories : celles de «Pacific peoples » ou «Tangata Pasifika» (les « gens du Pacifique » : tangata désignant les Hommes). Ce label est largement utilisé

7. Bien que cette estimation soit assez fréquemment donnée aujourd'hui, certains ont suggéré [Sutton et. al., 2008] qu'un petit groupe de personnes pourrait être arrivé avant cela. Néanmoins, ils suggèrent qu'une croissance démographique significative n'eut lieu que vers 800 ans. 
dans les médias et au sein des institutions néo-zélandaises : on parle ainsi de «Pasifika languages» à propos des langues austronésiennes parlées par les migrants du Pacifique, ces langues se voyant aujourd'hui incluses dans les programmes scolaires. Cette catégorie est également mobilisée dans les recensements de population et les statistiques démographiques. Ces différents éléments contribuent à l'émergence d'une définition institutionnelle et rationnelle des «Pacific peoples », qui masque l'hétérogénéité de ce groupe [Bedford, Didham, 2001, p. 28]. Les attitudes des migrants eux-mêmes par rapport à cette catégorie englobante sont variées, en Nouvelle-Zélande comme dans d'autres pays d'installation. En Australie par exemple, certains s'approprient ces catégories «pan-ethniques » de «Polynésien» ou «Pacific Islander» [Morton Lee, 2003, p. 245]. En Nouvelle-Zélande, selon C. McPherson [2001], les enfants d'immigrés du Pacifique ont plus facilement tendance à se définir comme des «Pacific person » que leurs parents. La migration aurait eu pour effet de faire passer les identifications d'un référent local (le village, l'île), à un référent national puis régional (l'identité Pacifique) [Ibid.]. Cependant, ce que H. Lee nomme les identités « ethno-nationales » (Tongien, Samoan, Cookien, etc.) demeurent significatives, y compris pour les descendants d'immigrés. T.K. Fitzgerald [1998] note ainsi que les jeunes originaires des îles Cook vivant en Nouvelle-Zélande s'identifient volontiers comme «Cook Islanders ». De même, les entretiens effectués par M. Anae [2001] auprès de descendants de migrants samoans nés en Nouvelle-Zélande montrent que l'identification comme «samoan » est forte, bien que les individus revendiquent aussi parfois, durant certaines périodes de leur vie, une identité de P.I. («Pacific Islander »). Selon elle, cette identification comme P.I. est un phénomène de jeunesse, qui tend à s'effacer au cours de la vie [Ibid., p. 112]. Les phénomènes migratoires et les processus d'identification et d'assignation des identités qui les accompagnent posent en outre des questions plus larges sur la définition de la société néo-zélandaise.

Officiellement, la Nouvelle-Zélande est un pays biculturel, vision qui repose sur l'idée que les Māori et les colons britanniques ont scellé un « pacte » fondant la Nouvelle-Zélande contemporaine, à travers la signature, en 1840, du traité de Waitangi. Celui-ci marquait, dans sa version anglaise du moins, le passage de la Nouvelle-Zélande sous souveraineté britannique tout en garantissant aux Māori qu'ils conserveraient autorité sur l'accès aux ressources naturelles et à la terre. Bien que contesté et bafoué [Kawharu, 1977 ; Belgrave, 2005], le traité fut ramené sur le devant de la scène dans les années 1970, pour servir une politique de réparation : il s'agissait, en se basant sur l'examen des violations dont le traité a été l'objet, de compenser les spoliations subies par la population autochtone, mais aussi d'affirmer le caractère biculturel de la Nouvelle-Zélande : les Māori étaient ainsi définis comme les « hôtes » et tous les groupes arrivés par la suite (que ce soit les colons européens ou les immigrés plus récemment), comme des « invités » [McIntosh, 2001, p. 151].

À cette vision biculturelle de la société s'oppose une vision multiculturelle, selon laquelle la Nouvelle-Zélande est composée non pas uniquement ou 
principalement de deux groupes fondateurs (Māori et Pākehā, c'est-à-dire descendants d'Européens), mais d'une multitude de communautés. Cette vision est notamment défendue par des immigrés, en particulier ceux issus de la région Pacifique : bien que sensibles à leur expérience commune de la discrimination et des inégalités sociales et à une certaine proximité culturelle avec les Māori, les immigrés du Pacifique et leurs descendants ont été réticents à épouser la cause du biculturalisme [Ibid.]. La vision multiculturaliste a également séduit des Pākehā, en particulier les leaders du National Party [Gagné, 2008], critiques face à la politique de réparation qui s'est traduite par la restitution à certains groupes Māori de biens et titres de propriété représentant une valeur se chiffrant souvent en millions de dollars ${ }^{8}$. Bien que fondées sur la reconnaissance des spécificités culturelles des « communautés » ou « minorités », les politiques multiculturalistes entrent donc en concurrence avec la vision biculturelle de la Nouvelle-Zélande et sont perçues par certains Māori comme une menace pour la reconnaissance de leur statut d'autochtone [McIntosh, 2001].

La circulation des pratiques esthétiques polynésiennes et les processus de patrimonialisation dont elles peuvent être l'objet doivent être examinés dans ce contexte. Les performances musicales et chorégraphiques de Polynésie, principalement introduites par les migrations, sont présentes dans diverses situations : le Polyfest ou «ASB Auckland Secondary Schools Maori and Pacific Islands Cultural Festival » et le Pasifika Festival en sont deux des principales occurrences. La première, manifestation organisée annuellement depuis 1976 dans un collège ou lycée d'Auckland a notamment pour objectif affiché de faire en sorte que les élèves (principalement māori ou enfants de migrants du Pacifique) puissent montrer la fierté que leur procurent leur identité culturelle et leur « patrimoine » (heritage). La seconde, non limitée aux scolaires, a pour objectif de rassembler les « communautés » du Pacifique. Les danses polynésiennes - en particulier des îles Cook, Tahiti, Hawai 'i - sont également présentes dans les écoles de danses privées et dans le système scolaire.

En revanche, la très grande majorité des performances «culturelles » effectuées pour un public de touristes internationaux en Nouvelle-Zélande sont fondées - contrairement à ce que l'on a vu à Tonga - sur des performances dansées qui font uniquement intervenir une démonstration de kapa haka, terme qui désigne les « arts vivants » māori, faits de musique, de danse et de poésie. Les auteurs de ces performances - dans leur grande majorité māori - refusent donc l'introduction de danses «polynésiennes » en mettant l'accent sur le caractère unique de la culture māori et sur le fondement «biculturel» de la nation néo-zélandaise.

Dans les institutions muséales ou patrimoniales en revanche, les kapa haka māori n'ont pas le monopole. Alors que le musée d'Auckland propose des

8. Ceci n'a pas empêché des alliances entre le National Party (situé à droite de l'échiquier politique) et le Māori Party pour former des gouvernements en 2008 et 2011, créant des dissensions à l'intérieur de ce dernier. 
performances de kapa haka à ses visiteurs, le musée national du Te Papa Tongarewa à Wellington (capitale de la Nouvelle-Zélande) avait, entre 2007 et 2008, fait un tout autre choix. L'institution proposait une performance (nommée Taonga MataOra) fondée sur un mélange de danses «polynésiennes »: les danses de Sāmoa, des Îles Cook et les kapa haka, occupaient chacune approximativement un tiers du temps de la performance, au cours d'une soirée incluant également un dîner. La performance dansée était une invitation à retracer l'histoire du peuplement de la Polynésie, en quelque sorte à «contre-courant», depuis la NouvelleZélande jusqu'à Sāmoa où, expliquait-on, étaient apparus les traits caractéristiques des cultures polynésiennes. Le guide de la soirée soulignait, tout en rappelant le statut particulier des Māori en Nouvelle-Zélande - en tant qu'autochtones - que ces derniers étaient avant tout des Polynésiens, ne se distinguant que peu des autres « Îliens du Pacifique » habitant la Nouvelle-Zélande.

Ces divers choix de présentation peuvent être mieux compris en se tournant vers le contexte plus général du musée [Condevaux, 2015]. Celui-ci est né en 1992 de la réunion du National Art Gallery et du National Museum. Le projet muséal - émergeant dans un contexte international de renouveau des pratiques muséographiques d'un côté [Gore, 2002 ; McCarthy, 2007] et le contexte national de renouveau culturel māori de l'autre - entendait notamment être plus représentatif des « minorités ». L'institution était donc organisée sur un principe biculturel, reflété dans l'architecture du bâtiment lui-même [McCarthy, 2007, p. 170], et qui reposait sur une plus grande implication des iwi Māori dans la préparation des expositions et les pratiques de conservation [Ibid.]. En même temps, l'une des « visées » affirmées du Te Papa est d'offrir une reconnaissance de l'importance des Māori, des Européens et des « autres traditions et patrimoines culturels » présents en Nouvelle-Zélande, dans le but de définir l'identité néo-zélandaise [Museum of New Zealand Te Papa Tongarewa, 2006, p. 5]. L'institution, comme tous les musées nationaux, ne pouvait donc échapper à des tensions importantes, entre d'un côté la reconnaissance de la diversité des rapports aux passés et de l'hétérogénéité des cultures, et de l'autre le fait que « the national museum exists primarily to tell the story of the nation, and in order to properly understand that story the museum inevitably needs to retain some kind of coherent narrative, to show the nation's progress and to hold all the other stories together. » [Gore, 2002, p. 305]. C'est dans cette logique que, par le biais de la performance évoquée ci-dessus, le Te Papa participe à la patrimonialisation de pratiques esthétiques dansées et chantées « polynésiennes » qui, on l'a vu, sont loin de se voir attribuer le même statut dans les institutions patrimoniales tongiennes ou dans les performances touristiques organisées par des Māori.

Dans le contexte du Musée Te Papa, les pratiques esthétiques polynésiennes sont incluses dans la définition d'un «patrimoine » national commun et d'une identité nationale «hybride», comme d'ailleurs dans certaines expositions du musée [Condevaux, 2015]. Elles constituent également pour les jeunes danseurs qui les exécutent le ciment d'une appartenance que l'on pourrait qualifier de panpolynésienne. Ces danseurs qui suivaient ou avaient suivi un cursus en «Pacific 
Performing Arts » étaient d'origines variées : l'un au moins de leurs parents, parfois les deux, était Māori ou originaire des îles Cook, de Sāmoa ou de Tonga. Au cours des entretiens, la plupart d'entre eux insistaient sur les similitudes entre les différentes sociétés de «Polynésie » et présentaient ces dernières comme formant une « grande famille» [Condevaux, 2015, p. 206-207].

Du point de vue de l'institution, cette requalification patrimoniale peut être envisagée comme une forme de reconnaissance accordée à l'importance de cette communauté des «Pacific Islanders » en Nouvelle-Zélande. En effet, comme le soulignent H. Bertheleu [2014] ou M. Rautenberg [2007], la reconnaissance patrimoniale est signe de reconnaissance sociale. Assurer une place aux « Pacific Islanders» au sein du musée serait signe d'une volonté de valoriser leur identité. Cependant, il est utile de rappeler que la « reconnaissance » procède nécessairement de rapports asymétriques entre celui qui reconnaît et celui qui tente d'être reconnu [Dousset, Préaud, 2014, p. 349]. Dans ce contexte, la « reconnaissance » risque de contribuer à une homogénéisation et une simplification de l'image de cette «communauté » des migrants du Pacifique, en réalité hétérogène. De plus, le choix, dans Taonga MataOra, de privilégier une vision multiculturelle de la société néo-zélandaise par rapport à une vision biculturelle peut être interprété comme résultant de la volonté de minimiser la place des Māori, comme le souligne D. Butts [2006] dans le cas du Whanganui Regional Museum, même si au Te Papa l'autochtonie de ces derniers était souvent rappelée [Condevaux, 2015].

\section{Conclusion}

La performance du musée du Te Papa comme les performances tongiennes qui ont une composition similaire font intervenir des danses de Polynésie occidentale (Sāmoa, Tonga), en particulier les danses assises caractéristiques de cette région, des danses de Polynésie orientale, caractérisées par ces déhanchés qui en font les danses les plus emblématiques de la Polynésie, et enfin les kapa haka māori. Alors qu'à Wellington, au musée du Te Papa, cette composition fait sens pour les danseurs et acteurs impliqués parce qu'elle met en scène ou donne corps à une identité «polynésienne » ou «pan-polynésienne » revendiquée par certains, à Tonga en revanche, où l'emprunt de danses polynésiennes est paradoxalement beaucoup plus fréquent, il ne correspond à aucune forme d'identification. Dans leur circulation, les pratiques esthétiques revêtent de nouvelles significations : d'emblèmes d'une identité localisée, liée à une appartenance territoriale à un village, une île ou un archipel, ces danses peuvent devenir les symboles d'une identité panpolynésienne revendiquée. Cela peut notamment s'expliquer par le contexte local de vie des acteurs concernés qui fréquentent au quotidien des personnes originaires d'autres archipels du Pacifique, parfois au sein de leur entourage familial.

Mais pour expliquer comment ces pratiques accèdent au statut de «patrimoine » reconnu par les institutions, il est nécessaire d'aller plus loin et de prendre en compte la spécificité des contextes locaux. À Tonga, pays d'émigration et non d'immigration, les politiques patrimoniales sont orientées par la volonté de 
construire une identité nationale fondée notamment sur la référence à la royauté. Dans ce contexte, les performances tongiennes seules accèdent au statut de «patrimoine ». En Nouvelle-Zélande, pays d'immigration, le rapport entre l'État et les « minorités »- autochtones ou issues de l'immigration - est historiquement ancré dans l'histoire coloniale et dans des relations de pouvoir déséquilibrées [Dousset, Préaud, 2014, p. 339]. La contextualisation du spectacle mentionné dans l'ensemble plus large des performances touristiques māori montre que ces emprunts sont minoritaires dans un contexte dans lequel l'affirmation de la spécificité de l'identité māori vis-à-vis des migrants du Pacifique est un enjeu politique fort pour préserver les acquis de la «renaissance māori» [Sissons, 1993 ; McIntosh, 2001]. La « reconnaissance » du patrimoine des migrants du Pacifique dans l'espace muséal national peut ainsi masquer d'autres enjeux politiques, et la non-reconnaissance simultanée d'un statut spécifique aux Māori autochtones.

\section{Bibliographie}

ADAms K. [2003], « Cultural displays and tourism in Africa and the Americas », Ethnohistory, vol. $50, \mathrm{n}^{\circ} 3$, p. 567-573.

AleXEYEFF K. [2009], Dancing from the heart. Movement, gender and Cook Islands globalization, Honolulu, University of Hawaii Press.

ANAE M. [2001], «The new "Viking of the Sunrise": New Zealand-borns in the information age », in MacPherson C., Spoonley P., Anae M. (dir.) Tangata o te Moana Nui. The evolving identities of Pacific peoples in Aotearoa/New Zealand, Palmerston North, Dunmore Press.

Asia/Pacific Cultural Centre for UNESCO (ACCU) [2008-2009], Case study report: New Zealand. A case study of Kapa haka, Ministry for culture and heritage, international partnership programme for safeguarding of intangible cultural heritage : www.accu.or.jp/ich/en/ training/casestudy_pdf/case_study_report_newzealand.pdf (page consultée le 17 mars 2015).

BEDFord R., Didham R. [2001], «Who are the "Pacific Peoples"? Ethnic identification and the New Zealand census », in MacPherson C., Spoonley P., Anae M. (dir.), Tangata o te Moana Nui. The evolving identities of Pacific peoples in Aotearoa/New Zealand, Palmerston North, Dunmore Press, p. 21-43.

Belgrave M. [2005], Historical frictions. Maori claims and reinvented histories, Auckland, Auckland University Press.

BERTHELEU H. (dir.) [2014], Au nom de la mémoire. Le patrimoine des migrations en région Centre, Tours, Presses universitaires François Rabelais.

Besnier N. [2011], On the edge of the global. Modern anxieties in a Pacific Island Nation, Stanford (Calif.), Stanford University Press.

ButTs D. [2006], « Museum governance, indigenous recognition and (in)tolerant multiculturalism », New Zealand Sociology, vol. 21, n 1, p. 89-107.

Chappell D. [1999], «Transnationalism in central oceanian politics : a dialectic of diasporas and nationhood? », Journal of the Polynesian Society, vol. 108, n 3, p. 277-304. 
Clavairolle F. [2014], « Faire le patrimoine. Introduction au processus contemporain de patrimonialisation », in BERTHELEU H. (dir.), Au nom de la mémoire. Le patrimoine des migrations en région Centre, Tours, Presses Universitaires François Rabelais.

Condevaux A. [2015], « All the same family ? Constructing and embodying the "Pacific Peoples" category in New Zealand », in Barbour S., Howard D., Misrahi-BaraK J., Lacroix T. (dir.), Diasporas, Cultures of Mobilities, "Race", vol. 2, Diaspora, Memory and Intimacy, Montpellier, Presses universitaires de la Méditerranée, p. 195-212.

Condevaux A. [2011], «Gender and power in Tongan tourist performances », Ethnology, vol. 50, n 3, p. 223-244.

Cook L., Didham R., Khawaja M. [2001], « The shape of the future : on the demography of Pacific People» in MacPherson C., Spoonley P., Anae M. (dir.), Tangata o te Moana Nui. The evolving identities of Pacific peoples in Aotearoa/New Zealand, Palmerston North, Dunmore Press, p. 44-65.

DOQUET A. [2003], Identité culturelle et tourisme: les mises en scène de l'authenticité culturelle en pays dogon et dans le Mande, Bamako, Centre Djoliba, compte rendu de communication: http://horizon.documentation.ird.fr/exldoc/pleins_textes/divers07/010032577 (page consultée le 19 novembre 2007).

Dousset L., Preaud M. [2014], « Reconnaissance ? Une critique anthropologique du cas australien », in Dousset L., Glowczewski B., Salaün M. (dir.), Les Sciences humaines et sociales dans le Pacifique Sud. Terrains, questions et méthodes, Marseille, Pacific-credo Publications, p. 333-357.

Dumont A. [2014], « Mémoires, territoires et mobilités », in Bertheleu H. (dir.), Au nom de la mémoire. Le patrimoine des migrations en région centre, Tours, Presses Universitaires François Rabelais, p. 85-90.

FitzGERALD T. K. [1998], «Metaphors, media and social change : second generation Cook Islanders in New Zealand » in WASSMANN J. (dir), Pacific answers to western hegemony: cultural practices of identity construction, New York (N. Y.), Oxford, Berg, p. 253-267.

Fourcade M.-B., Legrand C. (dir.) [2008], Patrimoines des migrations, migrations des patrimoines, Québec, Presses universitaires de Laval.

GAGNÉ N. [2008], «On the ethnicisation of New Zealand politics : the foreshore and seabed controversy in context », The Asia Pacific Journal of Anthropology, vol. 2, no 9, p. 123-140.

Gershon I. [2007], «Viewing diasporas from the Pacific: what Pacific ethnographies offer Pacific diaspora studies », The Contemporary Pacific, vol. 19, n 2, p. 474-502.

Gore M. [2002], Representations of history and nation in museums in Australia and Aotearoa New-Zealand. The National Museum of Australia and the Museum of New Zealand Te Papa Tongarewa, Melbourne, Université de Melbourne, Département d'histoire.

Goss J., LINDQUist B. [2000], «Placing movers : an overview of the Asian-Pacific migration system », The Contemporary Pacific, vol. 12, n 2, p. 385-414.

GOVERNMENT OF TONGa [1998], Report of the minister responsible for tourism for the year 1998, Nuku'alofa, Tonga, Tonga Visitors Bureau.

GRIJP (VAN DER) P. [2001], « Configurations d'identité et contextes coloniaux : une comparaison entre Tonga et Hawaii », Journal de la Société des Océanistes, n 113, p. 177-192.

Hermann E., KempF W., van MeiJl T. (dir.) [2014], Belonging in Oceania. Movement, Placemaking and multiple identifications, New York (N. Y.), Berghahn.

Johannson Fua S., Tuita T., Kanongata'A S. L., FuKo K. [2011], Cultural mapping, planning and policy: Tonga, Secretariat general of the Pacific community on behalf of the Ministry 
of Education, Women's Affairs and Culture, Nuku'alofa, Government of Tonga: www.spc.int/hdp/index2.php?option=com_docman\&task=doc_view\&gid=297\&Itemid=4 (page consultée le 23 novembre 2014).

KAEPPleR A. L. [1993], Poetry in motion : studies of tongan dance, Tongatapu, Vava'u Press.

KAEPPLER [2001], «Dance and the concept of style », Yearbook for Traditional Music, $\mathrm{n}^{\circ} 33$, p. 49-63.

KAWHARU I. H. [1977], Maori land tenure : studies of a changing institution, Oxford, Oxford University Press.

LAWRENCE H. R. [1992], "Is the "tahitian" drum dance really tahitian ? Re-evaluating the evidence for the origins of contemporary polynesian drum dance », Yearbook for Traditional Music, $\mathrm{n}^{\circ}$ 24, p. 126-137.

McCARTHY C. [2007], Exhibiting maori : a history of colonial cultures of display, Auckland, Te Papa Press.

McIntosh T. [2001], « Hibiscus in the Flax Bush : the Maori-Pacific Island interface » in MacPherson C., Spoonley P., Anae M. (dir.), Tangata o te Moana Nui. The evolving identities of Pacific peoples in Aotearoa/New Zealand, Palmerston North, Dunmore Press, p. 141-154.

MacPherson C. [2001], «One trunk sends out many branches : pacific cultures and cultural identities », in MacPherson C., Spoonley P., Anae M. (dir.), Tangata o te Moana Nui. The evolving identities of Pacific peoples in Aotearoa/New Zealand, Palmerston North, Dunmore Press, p. 66-85.

Mageo J. [2008], «Zones of ambiguity and identity politics in Samoa », Journal of the Royal Anthropological Institute, vol. 14, $\mathrm{n}^{\mathrm{0}} 1$, p. 61-78.

Morton LeE H. [2003], Tongans overseas. Between two shores, Honolulu, University of Hawai'i Press.

Moulin J. F. [1996], «What's mine is yours? Cultural borrowing in a Pacific context», Contemporary Pacific, vol. 8, $\mathrm{n}^{\circ}$ 1, p. 128-153.

Moyle R. [1991], Polynesian music and Dance, Auckland, University of Auckland, Centre for Pacific Studies.

Museum of New Zealand Te Papa Tongarewa [2006], Statement of intent 2006/2007 to 2008/2009: www.tepapa.govt.nz/SiteCollectionDocuments/AboutTePapa/Legislation Accountability/SOI2006.pdf (page consultée le 27 mars 2015).

NESPER L. [2003], «Simulating culture: being Indian for tourists in Lac du Flambeau's Wa-Swa-Gon Indian Bowl », Ethnohistory, vol. 50, n 3, p. 447-472.

RAUTENBERG M. [2007], «Les communautés imaginées de l'immigration dans la construction patrimoniale », Les Cahiers de Framespa, $\mathrm{n}^{\circ} 3$ : http://framespa.revues.org/274 (page consultée le 25 février 2013).

SCHWIMmer E. [1990], « The Maori hapu : a generative model », The Journal of the Polynesian Society, vol. 99, n 3, p. 297-317.

Sissons J. [1993], « The systematisation of tradition : Maori Culture as a strategic resource », Oceania, vol. 64, $\mathrm{n}^{\circ}$ 2, p. 97-116.

Statistics New Zealand [2013], Census ethnic group profile: www.stats.govt.nz/Census/ 2013-census/profile-and-summary-reports/ethnic-profiles.aspx?request_value=24652\&parent_ id=24650\&tabname=\#24652 (page consultée le 3 mars 2016). 
Statistics New Zealand [2013], Census quickStats about culture and identity, www.stats.govt.nz/Census/2013-census/profile-and-summary-reports/quickstats-cultureidentity/pacific-peoples.aspx (page consultée le 3 mars 2016).

Stillman A. K. [1999], « Globilizing Hula », Yearbook for traditional music, nº 31, p. 57-66.

Stillman A. K. [1988], «Images and realities : visitors' responses to tahitian music and dance » in Come Mek Me Hol' Yu Han': the impact of tourism on traditional music, Kingstone, Jamaica Memory Bank, p. 145-66.

Sutton D., Flenley J., Li X., Todd A., Butler K., Summer R., Chester P. I. [2008], « The timing of human discovery and colonization of New Zealand », Quaternary International, $\mathrm{n}^{\circ} 184$, p. $109-121$.

TCherkezoff S. [2007], Polynésie/Mélanésie. L'invention française des « races » et des régions de l'Océanie (XVI'-XIXe siècles), Tahiti, Au vent des Îles.

TEILHET-Fisk J. [1996], « The Miss Heilala beauty pageant: where beauty is more than skin deep », in COHEN C. B., Wilk R., Stoeltje B. (dir.), Beauty queens on the global stage : gender, contests, and power, London, Routledge, p. 185-202.

Torgersen E. H. [2010], The social meanings of hula. Hawaiian traditions and politicized identities in Hilo, thèse soumise pour l'obtention partielle du diplôme de M.A., département d'anthropologie sociale, université de Bergen: https://bora.uib.no/bitstream/handle/ 1956/4593/72247037.pdf?sequence=1 (page consultée le 3 avril 2015).

Unesco [2010], Comité du patrimoine mondial, $34^{e}$ section, rapport sur les propositions d'inscription et les biens en série: http://whc.unesco.org/archive/2010/whc10-34Com-9Bf.pdf (page consultée le 7 décembre 2013).

'EsAu R. L. [2005], «Tongan immigrants in New Zealand», Asian and Pacific Migration Journal, vol. 14, nº 4, p. 441-466. 